Trinity University

Digital Commons@ Trinity

Mathematics Faculty Research

Mathematics Department

$6-2011$

\title{
Budding Yeast, Branching Processes, and Generalized Fibonacci Numbers
}

Peter Olofsson

Trinity University, polofsso@trinity.edu

Ryan C. Daileda

Trinity University, Ryan.Daileda@trinity.edu

Follow this and additional works at: https://digitalcommons.trinity.edu/math_faculty

Part of the Mathematics Commons

\section{Repository Citation}

Olofsson, P., \& Daileda, R.C. (2011). Budding yeast, branching processes, and generalized Fibonacci numbers. Mathematics Magazine, 84(3), 163-172. doi: 10.4169/math.mag.84.3.163

This Post-Print is brought to you for free and open access by the Mathematics Department at Digital Commons @ Trinity. It has been accepted for inclusion in Mathematics Faculty Research by an authorized administrator of Digital Commons @ Trinity. For more information, please contact jcostanz@trinity.edu. 


\title{
Budding yeast, branching processes, and generalized Fibonacci numbers
}

\author{
Peter Olofsson* and Ryan C. Daileda ${ }^{\dagger}$
}

September 14, 2010

\begin{abstract}
A real-world application of branching processes to a problem in cell biology where the generalized Fibonacci numbers known as $k$ nacci numbers play a crucial role is described. The $k$-nacci sequence is used to obtain asymptotics, computational formulas, and to justify certain practical simplifications. Along the way, an explicit formula for the sum of $k$-nacci numbers is established.
\end{abstract}

Keywords: Branching process, yeast, $k$-nacci sequence, Fibonacci numbers, linear recurrence AMS 2000 Mathematics Subject Classification Codes: 11B39, 60J80, 62P10, 92D25

\section{Introduction}

The Fibonacci sequence is famous for showing up in nature in a multitude of ways, many of which are however idealized such as Fibonacci's famous medieval breeding rabbits. In Olofsson and Bertuch (2010), a branching process model was used to analyze experiments on growing yeast populations and it turned out that the generalized Fibonacci numbers known as " $k$-nacci

\footnotetext{
${ }^{*}$ Corresponding author. Trinity University, Mathematics Department, One Trinity Place, San Antonio, TX 78212. Email:polofsso@trinity.edu

${ }^{\dagger}$ Trinity University, Mathematics Department, One Trinity Place, San Antonio, TX 78212.
} 
numbers" were crucial to practical results and calculations. In this paper, we elaborate on some of the mathematics that were not fully developed in Olofsson and Bertuch, the focus of that paper being to model and analyze a problem in biology. The aim of the present paper is to give a nice example of how $k$-nacci numbers may quite unexpectedly show up in a practical problem and how number theoretical results may be of significant practical use.

\section{Budding yeast}

The yeast Saccharomyces cerevisiae is not only of great commercial value for its use in baking and brewing, it is also one of the most important model organisms in biology. It is a unicellular organism that reproduces through budding, meaning that a yeast cell reproduces by a new yeast cell starting to grow on its surface, eventually separating from its mother as a newborn daughter cell. This reproduction scheme is different from that of binary fission, common in many bacteria such as E. Coli, where the cell grows to divide into two new cells of equal size. Although both reproductions schemes give rise to clones, that is, cells that are genetically identical (save for mutations), there are differences that matter to the mathematical modeling as we shall see in the next section.

Since yeast has linear chromosomes just like human beings, its genetics can be studied for greater insight into human genetics. One example thereof is the study of the shortening of chromosomal ends known as telomeres that is known to take place in many of our cells. With each cell division, telomeres become progressively shorter until they reach a point at which the cell stops dividing, to avoid damage to the coding DNA in the interior of the chromosome. Such a nondividing cell is said to be senescent. To counteract telomere shortening, some cells, for example embryonic stem cells, contain the enzyme telomerase which adds telomere sequences so that the chromosomes can maintain a stable telomere length. As it turns out, some cells manage to keep replicating even without telomerase; such populations were studied in Olofsson and Bertuch (2010), using branching processes to model populations of yeast cells and to estimate cell population parameters from laboratory data on such cells. 


\section{Branching processes}

A branching process is a stochastic model for a proliferating population where assumptions are made on individual reproduction to draw conclusions about population behavior. One of the simplest cases is to consider a population of cells that reproduce by binary splitting after going through the cell cycle. Thus, an individual always gives rise to two daughters and randomness enters via the cell cycle times (or "lifetimes") which are assumed to be independent random variables with common cumulative distribution function (cdf) $F$. Start such a population from one individual and let $M(t)$ denote the expected number of individuals at time $t$.

To arrive at an expression for $M(t)$, first note that there are $2^{n}$ cells in the $n$th generation. Such a cell is present at time $t$ if the sum of $n$ cell cycle times is less than $t$ and the sum of $n+1$ cell cycle times is greater than $t$. The sum of $j$ cell cycle times has a cdf that is the $j$-fold convolution of $F$ with itself, denoted $F^{* j}$. Thus, the probability that an $n$ th-generation cell is present at time $t$ equals $F^{* n}(t)-F^{*(n+1)}(t)$ and the expected number of cells in generation $n$ that are present at time $t$ equals

$$
2^{n}\left(F^{* n}(t)-F^{*(n+1)}(t)\right)
$$

and summing over all generations gives the expression

$$
M(t)=1-F(t)+\sum_{n=1}^{\infty} 2^{n}\left(F^{* n}(t)-F^{*(n+1)}(t)\right)
$$

where the first term $1-F(t)$ accounts for the ancestral cell which by definition is in generation $n=0$. By convention, a 0 -fold convolution is defined as a unit point mass at 0 so that $F^{* 0}(t) \equiv 1$, and since $2^{0}=1$ we can write $M(t)$ more compactly as

$$
M(t)=\sum_{n=0}^{\infty} 2^{n}\left(F^{* n}(t)-F^{*(n+1)}(t)\right)
$$

Note that we assume that all cells survive to reproduce. If we instead assume that each cell survives to reproduce with probability $p$, otherwise dies without reproducing, the mean number of offspring per cell is $m=2 p$ where we assume that $p>1 / 2$ so that $m>1$ which gives a population that, on average, increases in size. We get instead the expression 


$$
M(t)=\sum_{n=0}^{\infty} m^{n}\left(F^{* n}(t)-F^{*(n+1)}(t)\right)
$$

Adopt the standard notation $x(t) \sim y(t)$ if $x(t) / y(t) \rightarrow 1$ as $t \rightarrow \infty$. The asymptotic growth rate of $M(t)$ is then given by

$$
M(t) \sim b e^{\alpha t} \text { as } t \rightarrow \infty
$$

where the constants $\alpha$ and $b$ are determined by the lifetime cdf $F$ and the mean number of offspring per individual $m$. Specifically, let

$$
\widehat{F}(s)=\int_{0}^{\infty} e^{-s t} F(d t)
$$

the Laplace transform of the probability measure associated with $F$. The growth rate $\alpha$, called the Malthusian parameter, is defined through the relation

$$
m \widehat{F}(\alpha)=1
$$

where $m>1$ implies that $\alpha>0$ (in our model, $m=2$ ). The constant $b$ can be shown to equal

$$
b=\left(4 \alpha \int_{0}^{\infty} t e^{-\alpha t} F(d t)\right)^{-1}
$$

and we refer to Harris (1963) or Jagers and Nerman (1984) for proofs and further details.

However, as pointed out earlier, yeast does not reproduce by binary splitting but rather by budding. Starting from one cell, after the first budding event there are two cells and although one is the mother and the other is the daughter, we can still refer to the pair as the "first generation." In this sense there is no difference from binary splitting but it is known that a mother cannot give birth to an unlimited number of daughters. Thus, rather than $2^{n}$ cells in the $n$th generation, we get a number $m(n)$ and the expression

$$
M(t)=\sum_{n=0}^{\infty} m(n)\left(F^{* n}(t)-F^{*(n+1)}(t)\right)
$$

Growth rate and other asymptotic properties of $M(t)$ are now determined by $F$ together with $m(n)$. For applications, we need to compute $M(t)$ for finite $t$ which requires getting a handle on the number $m(n)$. We address this task in the next section. 


\section{Generalized Fibonacci numbers}

By the $k$-nacci sequence we mean the sequence $\left\{F_{j}, j \geq 0\right\}$ defined by $F_{0}=$ $F_{1}=\ldots=F_{k-2}=0, F_{k-1}=1$, and $F_{n}=F_{n-1}+F_{n-2}+\ldots+F_{n-k}$ for $n \geq k$. For example, when $k=3$, each term is the sum of the three previous terms:

$$
0,0,1,1,2,4,7,13,24, \ldots
$$

In the yeast population, suppose that a mother cell can have $k$ daughter cells before she stops reproducing (in biology, $k$ is known as the proliferative lifespan). In any given generation $n$, cells can be divided into classes describing how many more daughter cells they can have. Thus, let $N_{j}^{(n)}$ be the number of cells in generation $n$ that can have an additional $j$ daughter cells for $j=0,1, \ldots, k$. The class with $j=0$ is the class of senescent cells, and we assume that they stay in the population indefinitely (although it is easy to model a scenario where they eventually die and disappear). The class with $j=k$ are the newborn cells that have yet to reproduce. As it turns out, the numbers of cells in these classes are precisely described by the $k$-nacci sequence.

Proposition 4.1 Consider the vector $\left(N_{0}^{(n)}, N_{1}^{(n)}, \ldots, N_{k}^{(n)}\right)$ in the $n$th generation of the branching process above. Let $F_{i}$ denote the ith $k$-nacci number and let $S_{n}=F_{0}+F_{1}+\ldots+F_{n}$. Then $\left(N_{0}^{(n)}, N_{1}^{(n)}, \ldots, N_{k}^{(n)}\right)$ equals

$$
\left(S_{n-1}, F_{n}, F_{n+1}, \ldots, F_{n+k-1}\right)
$$

Proof. In generation 0 there is one cell that is able to divide $k$ more times which gives the vector $(0,0, \ldots, 0,1)$ for generation 0 . Each cell with $j \geq 1$ produces a daughter cell that is able to reproduce $k$ times and is then itself able to reproduce another $j-1$ times. Cells with $j=0$ remain unchanged. Thus, each class with $j \geq 1$ feeds into the class $j-1$ immediately below it, and also into the highest class $k$. The transition from generation $n-1$ to generation $n$ can be described as follows: 


$$
\left\{\begin{array}{l}
N_{0}^{(n)}=N_{0}^{(n-1)}+N_{1}^{(n-1)} \\
N_{j-1}^{(n)}=N_{j}^{(n-1)} \text { for } 2 \leq j \leq k \\
N_{k}^{(n)}=\sum_{j=1}^{k} N_{j}^{(n-1)}
\end{array}\right.
$$

and the proposition follows.

The total number of cells in the $n$th generation, $m(n)$, equals

$$
\begin{aligned}
m(n) & =S_{n-1}+F_{n}+\ldots+F_{n+k-1} \\
& =S_{n+k-1}
\end{aligned}
$$

and Proposition 4.1 provides a recursive scheme that enables us to compute $m(n)$. For example, if $k=4$, the first terms in the sequence $\{m(n), n \geq 0\}$ are

$$
1,2,4,8,16,31,60,116,224, \ldots
$$

where we recognize the powers of 2 until the 4 th generation $(n=4)$ after which the effect of the proliferative lifespan $k=4$ becomes noticeable and slows down the growth. By (3.5), we can also compute the expected number $M(t)$ of cells at each time $t$ which enables us to compare the model with laboratory data and estimate unknown parameters. As it turns out, we can even get an explicit expression for $m(n)$, expressed in terms of $k$-nacci numbers which is crucial to establish asymptotics of the branching process. In the next section, we study the $k$-nacci numbers as a special case of linear recurrence.

\section{Linear recurrences}

Given a positive integer $k$, and complex numbers $a_{0} \neq 0, a_{1}, \ldots, a_{k-1}$ consider the $k$-term linear recurrence

$$
R_{n}=a_{k-1} R_{n-1}+a_{k-2} R_{n-2}+\cdots+a_{0} R_{n-k} .
$$


By a solution to the recurrence (5.1) we mean a sequence $\left\{R_{n}\right\}_{n=0}^{\infty}$ whose values satisfy (5.1) for all $n \geq k$. Define the characteristic polynomial of (5.1) to be

$$
p(x)=x^{k}-a_{k-1} x^{k-1}-a_{k-2} x^{k-1}-\cdots-a_{0} .
$$

It is well known that the set of solutions to (5.1) forms a complex vector space. In fact, if (5.2) has distinct roots $r_{1}, r_{2}, \ldots, r_{k}$, the sequences $\left\{r_{j}^{n}\right\}_{n=0}^{\infty}$, $j=1,2, \ldots, k$ form a basis for this solution space. Put more concretely, if we are given initial values $R_{0}, R_{1}, \ldots, R_{k-1}$ and define $\left\{R_{n}\right\}_{n=0}^{\infty}$ recursively through (5.1), then there are unique complex coefficients $b_{1}, b_{2}, \ldots, b_{k}$ so that

$$
R_{n}=b_{1} r_{1}^{n}+b_{2} r_{2}^{n}+\cdots+b_{k} r_{k}^{n}
$$

for all $n \geq 0$, for details see Elaydi, 2005 .

Given a solution $\left\{R_{n}\right\}_{n=0}^{\infty}$ to $(5.1)$, we let $S_{n}=R_{0}+R_{1}+\cdots R_{n}$ for $n \geq 0$. The following proposition establishes a closed form expression for $S_{n}$.

Proposition 5.1 Consider the linear recurrence in (5.1). Assume that the characteristic polynomial has $k$ distinct roots, none of which equals 1 . Then there exist constants $c_{0}, \ldots, c_{k-1}$ such that

$$
S_{n}=\sum_{l=0}^{k-1} c_{l} R_{n+l+1}-\sum_{l=0}^{k-1} c_{l} R_{l}
$$

Proof. Express $R_{n}$ as a linear combination of the roots of (5.2), as in (5.3). Then we have

$$
\begin{aligned}
S_{n} & =\sum_{i=0}^{n} R_{i} \\
& =\sum_{i=0}^{n} \sum_{j=1}^{k} b_{j} r_{j}^{i} \\
& =\sum_{j=1}^{k} b_{j} \sum_{i=0}^{n} r_{j}^{i} \\
& =\sum_{j=1}^{k} b_{j} \frac{r_{j}^{n+1}-1}{r_{j}-1}
\end{aligned}
$$

Since the characteristic polynomial $p(x)$ does not have 1 as a root, $p(x)$ and $x-1$ are relatively prime so that we can find polynomials $u(x)$ and $v(x)$ which satisfy

$$
v(x) p(x)+u(x)(x-1)=1
$$


Moreover, by using the division algorithm if necessary, we can assume that $\operatorname{deg} u(x)<k$. Substituting any of the roots $r_{j}$ of $p(x)$ into (5.4) immediately yields

$$
\frac{1}{r_{j}-1}=u\left(r_{j}\right)
$$

It now follows from our work above that

$$
S_{n}=\sum_{j=1}^{k} b_{j}\left(r_{j}^{n+1}-1\right) u\left(r_{j}\right)
$$

If we write $u(x)=c_{k-1} x^{k-1}+\cdots+c_{0}$ this becomes

$$
\begin{aligned}
S_{n} & =\sum_{l=0}^{k-1} c_{l} \sum_{j=1}^{k} b_{j}\left(r_{j}^{n+l+1}-r_{j}^{l}\right) \\
& =\sum_{l=0}^{k-1} c_{l}\left(R_{n+l+1}-R_{l}\right) \\
& =\sum_{l=0}^{k-1} c_{l} R_{n+l+1}-\sum_{l=0}^{k-1} c_{l} R_{l} .
\end{aligned}
$$

which concludes the proof.

In the expression for $S_{n}$, note that the first sum includes at most $k$ terms of the sequence $\left\{R_{n}\right\}_{n=0}^{\infty}$, while the second sum depends only on the initial conditions $R_{0}, R_{1}, \ldots, R_{k-1}$.

As an example, we apply this result to the Fibonacci numbers, which are simply the $k=2$ case of the $k$-nacci numbers. The characteristic polynomial in this case is $p(x)=x^{2}-x-1$, which satisfies $-p(x)+x(x-1)=1$. Hence, $u(x)=x$ so that Proposition 5.1 becomes the familiar result $S_{n}=$ $F_{n+2}-F_{1}=F_{n+2}-1$.

As a corollary we obtain the corresponding result for the $k$-nacci numbers.

Corollary 5.2 For the $k$-nacci sequence $\left\{F_{j}, j \geq 0\right\}$, let $S_{n}=F_{0}+F_{1}+$ $\cdots+F_{n}$. Then

$$
S_{n}=\frac{1}{k-1}\left(F_{n+k}-\sum_{l=0}^{k-3}(k-l-2) F_{n+l+1}-1\right)
$$


Proof. To get an expression for the polynomial $u(x)$ which determines the coefficients $c_{l}$ in Proposition 5.1, note that for $k \geq 2$ we have

$$
\begin{aligned}
1 & =\frac{-1}{k-1}\left(x^{k}-x^{k-1}-\cdots-1\right) \\
& +\frac{1}{k-1}\left(x^{k-1}-x^{k-3}-2 x^{k-4}-\cdots-(k-2)\right)(x-1)
\end{aligned}
$$

which identifies $u(x)$ as

$$
u(x)=\frac{1}{k-1}\left(x^{k-1}-\sum_{l=0}^{k-3}(k-l-2) x^{l}\right),
$$

provided we treat the sum as empty when $k=2$. Clearly the characteristic polynomial $p(x)$ does not have 1 as a root. Regarding the distinctness of the roots of $p(x)$ observe that

$$
\begin{aligned}
& p(x)=x^{k}-\left(x^{k-1}+x^{k-2}+\cdots+1\right) \\
& =x^{k}-\frac{x^{k}-1}{x-1} \\
& =\frac{x^{k+1}-2 x^{k}+1}{x-1}
\end{aligned}
$$

and the polynomial in the numerator has no repeated roots, as it does not share any roots with its derivative (see Gallian, 2010). Finally, since $F_{0}=F_{1}=\cdots=F_{k-2}=0$ and $F_{k-1}=1$, the result follows from Proposition 5.1 .

\section{Asymptotics of the branching process}

The asymptotic results in this section rely on the fact that $k$-nacci num-

bers have asymptotic geometric growth. Following Flores (1967), there exist numbers $r$ and $A$ such that 


$$
F_{j} \sim A r^{j}
$$

as $j \rightarrow \infty$, meaning that $F_{j} / r^{j} \rightarrow A$ as $j \rightarrow \infty$. The number $r$ is the dominant root of the characteristic equation

$$
x^{k}-x^{k-1}-\cdots-x-1=0
$$

and it is known to be real and to lie between the golden ratio $\phi \approx 1.618$ and 2. In fact, for $k=2, r=\phi$ and as $k \rightarrow \infty, r \uparrow 2$. The constant $A$ equals

$$
A=\frac{r-1}{(k+1) r^{k}-2 k r^{k-1}}
$$

Next, we establish the long-term composition of cells in the different classes, recalling that class $j$ contains cells that can have an additional $j$ daughter cells, $j=0,1, \ldots, k$.

Proposition 6.1 Let $r$ be as above. The asymptotic proportions of cells in the classes $(0,1, \ldots, k)$ equal

$$
\left(r^{-k},(k-1) r^{-2 k+1},(k-1) r^{-2 k+2}, \ldots,(k-1) r^{-2 k+j+1}\right)
$$

Proof. By Proposition 4.1, the proportions equal

$$
\left(\frac{S_{n-1}}{S_{n+k-1}}, \frac{F_{n}}{S_{n+k-1}}, \ldots, \frac{F_{n+k-1}}{S_{n+k-1}}\right)
$$

for $j=1, \ldots, k$ and the proposition follows from Corollary 5.2 and (6.1).

Proposition 6.1 is not just a theoretical limit result, it has important practical implications for the yeast cell population studies. Certain computational expressions become greatly simplified if the finite proliferative lifespan can be neglected, instead assuming that each cell can produce an unlimited number of daughter cells. Since the fraction of senescent cells in a given generation $n$ is roughly $r^{-k}$, this number can be used to justify such an approximation. For example, for the regular Fibonacci sequence with $k=2$, we have $r=\phi$ and since $\phi^{-2} \approx 0.38$, as many as $38 \%$ of cells have reached the end of their proliferative lifespan and are no longer able to produce daughter cells (notice that also the fraction of newborns equals 38\%). In this case, the 
approximation would not work very well. Note that, since $r \uparrow 2$ as $k \rightarrow \infty$, the " $\infty$-nacci" sequence has $r=2$ and corresponds to a binary splitting branching process where each individual can produce an unlimited number of offspring.

For yeast cells, the proliferative lifespan $k$ has been estimated to be on average 25, Sinclair et al. (1998), which gives a value of $r$ that for all practical purposes equals 2 and the fraction of senescent cells is less than one in 10 million. For any reasonable duration of a yeast cell experiment, this fraction is negligible although it does of course matter to the theoretical asymptotic limits. The number 25 is an experimentally determined average and the true range may well go lower. However, calculations show that $r$ exceeds 1.99 already for $k=7$ in which case less than $1 \%$ of cells are senescent. For the yeast experiments considered in Olofsson and Bertuch (2010), $k$ is likely to largely exceed 7 and the approximation works well.

Finally, we obtain the asymptotic growth rate of $M(t)$. As we have seen above, $m(n)$ grows asymptotically as $r^{n}$, that is, like a branching process with mean number of offspring equal to $r$. Since $r=2 p$, the latter process is a binary splitting process where each cell survives to reproduce with probability $r / 2$, and dies without reproducing with probability $1-r / 2$. The next result shows that the Malthusian parameter is the same for the budding process with generation sizes $m(n)$ and the binary splitting process with mean $r$, but that the former process always has a larger expected value.

Corollary 6.2 Ast $\rightarrow \infty, M(t) \sim C b e^{\alpha t}$ where $\alpha$ and $b$ are as in (3.3) with mean number of offspring $m=r$. The constant $C$ depends on $k$ and satisfies $C>1$ and $C \rightarrow 1$ as $k \rightarrow \infty$.

Proof. Since $m(n)=S_{n+k-1}$, Corollary 5.2 and (6.1) yield

$$
m(n) \sim C r^{n}
$$

where

$$
C=\frac{A}{k-1}\left(r^{2 k-1}-\sum_{l=0}^{k-3}(k-l-2) r^{k+l}\right)
$$

$A$ being the constant defined in (6.2). If we, informally, substitute this expression for $m(n)$ in (3.5), we get 


$$
M(t) \sim C \sum_{n} r^{n}\left(F^{* n}(t)-F^{*(n+1)}(t)\right)
$$

as $t \rightarrow \infty$. By (3.3) and (3.4), we get

$$
M(t) \sim C b e^{\alpha t}
$$

as $t \rightarrow \infty$, as desired.

To prove that the substitution leading to (6.4) is indeed legitimate, let us formally prove that

$$
e^{-\alpha t} M(t) \rightarrow C b
$$

as $t \rightarrow \infty$. recall that $m(n) \sim C r^{n}$, that is, $m(n) / r^{n} \rightarrow C$ as $n \rightarrow \infty$. Choose $N$ such that

$$
C-\epsilon \leq \frac{m(n)}{r^{n}} \leq C+\epsilon
$$

for $n>N$. For ease of notation, let

$$
P(n, t)=F^{* n}(t)-F^{*(n+1)}(t)
$$

for $n=0,1,2, \ldots$ and note that $0 \leq P(n, t) \leq 1$ for all $n$ and $t$. We now get

$$
\begin{aligned}
& e^{-\alpha t} M(t)=e^{-\alpha t} \sum_{n=0}^{\infty} m(n) P(n, t) \\
& =e^{-\alpha t} \sum_{n=0}^{N} m(n) P(n, t)+e^{-\alpha t} \sum_{n=N+1}^{\infty} m(n) P(n, t)
\end{aligned}
$$

where the first term goes to 0 as $t \rightarrow \infty$ and hence the limit of $e^{-\alpha t} M(t)$ is the same as that of the second term, for which we have

$$
(C-\epsilon) e^{-\alpha t} \sum_{n=N+1}^{\infty} r^{n} P(n, t) \leq e^{-\alpha t} \sum_{n=N+1}^{\infty} m(n) P(n, t) \leq(C+\epsilon) e^{-\alpha t} \sum_{n=N+1}^{\infty} r^{n} P(n, t)
$$

Let $t \rightarrow \infty$ and use (3.4) to obtain

$$
(C-\epsilon) b \leq \lim _{t \rightarrow \infty} e^{-\alpha t} M(t) \leq(C+\epsilon) b
$$


Since $\epsilon$ was arbitrary, we conclude that

$$
\lim _{t \rightarrow \infty} e^{-\alpha t} M(t)=C b
$$

To prove that $C>1$, note that by (6.2) and (6.3), the constant $C$ equals

$$
C=\frac{A}{k-1}\left(r^{2 k-1}-\sum_{l=0}^{k-3}(k-l-2) r^{k+l}\right)
$$

which, after some algebra, simplifies to

$$
C=\frac{r\left(r^{k+1}-2 r^{k}+k r-k-r+2\right)}{(r-1)(k r+r-2 k)(k-1)}
$$

From (5.6) we conclude that

$$
r^{k+1}-2 r^{k}-1=0
$$

which simplifies $C$ further, to

$$
C=\frac{r(k r-k-r+1)}{(r-1)(k r+r-2 k)(k-1)}
$$

Simple but tedious calculations yield that $C>1$ if and only if

$$
\left(3 r-r^{2}-2\right)(k-1)>0
$$

where the first factor is positive for $1<r<2$ and since our $r$ is in this range, we conclude that $C>1$. Analyzing the numerator in (5.6) yields the inequalities

$$
2-\frac{1}{2^{k-1}}<r<2-\frac{1}{2^{k}}
$$

and more simple but tedious calculations reveal that $C \rightarrow 1$ as $k \rightarrow \infty$.

Corollary 6.2 shows that the branching process for budding yeast with proliferative lifespan $k$, asymptotically grows similarly to a binary splitting process with mean number of daughter cells equals to $r$, in the sense of having the same Malthusian parameter $\alpha$. However, since the binary splitting population grows as $b e^{\alpha t}$ and the budding population as $C b e^{\alpha t}$ where $C>1$, the budding process tends to be, on average, larger than the splitting process. Also note that the binary splitting population can go extinct which is not 
possible for the budding population. As $k \rightarrow \infty, C \rightarrow 1$ and $r \uparrow 2$ so in the limit, budding and binary splitting are equivalent which makes intuitive sense.

\section{Acknowledgments}

Peter Olofsson was funded by NIH research grant 1R15GM093957-01.

\section{References}

[1] S. Elaydi, An Introduction to Difference Equations. Springer, New York, 2005 .

[2] I. Flores, Direct calculation of k-generalized Fibonacci numbers. The Fibonacci Quarterly 5 (1967), 259-266

[3] J. Gallian, Contemporary Abstract Algebra. Brooks/Cole, Belmont, CA, 2010 .

[4] T.E. Harris, The Theory of Branching Processes. Springer, Berlin, 1963.

[5] P. Jagers and O. Nerman, The Growth and Composition of Branching Populations. Adv. Appl. Prob. 16 (1984), 221-259

[6] P. Olofsson and A.A. Bertuch, Modeling growth and telomere dynamics in S. cerevisiae. J. Theor. Biol. 263(3) (2010), 353-359

[7] D.A. Sinclair, K. Mills and L. Guarente, Aging in Saccharomyces cerevisiae. Annu. Rev. Microbiol. 52 (1998) , 533-560 